\title{
Comparative simulations for solutions of fractional Sturm-Liouville problems with non-singular operators
}

\author{
Erdal Bas $^{1 *}$ (D, Ramazan Ozarslan', Dumitru Baleanu ${ }^{2,3}$ and Ahu Ercan ${ }^{1}$
}

"Correspondence:

erdalmat@yahoo.com

'Department of Mathematics, Firat

University, Elazig, Turkey

Full list of author information is

available at the end of the article

\begin{abstract}
In this study, we consider fractional Sturm-Liouville (S-L) problems within non-singular operators. A fractional S-L problem with exponential and Mittag-Leffler kernels is given with different versions in the Riemann-Liouville and Caputo sense. Also, we obtain representation of solutions for $S-L$ problems by the Laplace transform and find analytical solutions of the problems. Finally, we compare the solutions of the problem with these different versions, and we also compare the solutions of the problem with exponential and Mittag-Leffler kernels together by simulation under different potentials, different orders, and different eigenvalues.
\end{abstract}

MSC: 34B24; 44A10; 34A08

Keywords: Sturm-Liouville; Fractional operator; Exponential kernel; Mittag-Leffler kernel; Laplace transform

\section{Introduction}

Fractional calculus has a lot of application areas in engineering, nature sciences, and mathematics. This range of application areas gave rise to new fractional definitions, especially for the real world modelling problems. More recently, several new fractional derivative definitions have been studied. One of those definitions is fractional derivative with exponential kernel defined by Caputo and Fabrizio [1], and another definition is fractional derivative with Mittag-Leffler kernel defined by Atangana and Baleanu [2]. Fractional operator with Mittag-Leffler kernel is a general form of the operator with exponential kernel because of $\alpha$ order in its definition. These new definitions enable more suitable results for some modelling problems in the real world because of having nonsingularity in the kernels.

Baleanu et al. [3-7], Atangana et al. [8, 9], and Abdeljawad et al. [10, 11] studied a fractional operator with exponential kernel for some modelling problems and their solution methods. After the emergence of the definition of fractional derivative with MittagLeffler kernel by Atangana and Baleanu [2], many scientists studied this operator [12-24]. Baleanu et al. [25-27], Atangana et al. [28, 29] studied the operator with Mittag-Leffler kernel. Many other scientists studied comparisons of these two new definitions, see Abro et al. [30], Sheikh et al. [31, 32], Saad et al. [33], and Gomez et al. [34].

(c) The Author(s) 2018. This article is distributed under the terms of the Creative Commons Attribution 4.0 International License (http://creativecommons.org/licenses/by/4.0/), which permits unrestricted use, distribution, and reproduction in any medium, provided you give appropriate credit to the original author(s) and the source, provide a link to the Creative Commons license, and indicate if changes were made. 
The emergence of Sturm-Liouville operators began as a one-dimensional Schrödinger equation in quantum mechanics. Fractional Sturm-Liouville differential equations were studied by Klimek et al. [35], Bas et al. [36, 37], Zayernouri et al. [38], Khosravian et al. [39], and Dehghan et al. [40].

In this study, we consider fractional Sturm-Liouville (S-L) problems within nonsingular operators. A fractional S-L problem with exponential and Mittag-Leffler kernels is given with different versions in the Riemann-Liouville and Caputo sense. Also, we obtain the representation of solutions for $\mathrm{S}-\mathrm{L}$ problems by the Laplace transform and find the analytical solutions of the problems. We analyze solutions of these different versions and display them by simulation under different potentials, different orders, and different eigenvalues. However, we compare the solutions of the problem with these different versions, and we also compare the solutions of the problem with exponential and MittagLeffler kernels together by simulation.

\section{Preliminaries}

Definition 1 ([1]) Fractional derivative with exponential kernel is defined as follows: left and right derivatives in the Caputo sense

$$
\begin{aligned}
& { }_{a}^{C F C} D^{\alpha} f(t)=\frac{M(\alpha)}{1-\alpha} \int_{a}^{t} f^{\prime}(s) \exp \left(\frac{-\alpha}{1-\alpha}(t-s)\right) d s, \\
& { }^{C F C} D_{b}^{\alpha} f(t)=\frac{-M(\alpha)}{1-\alpha} \int_{t}^{b} f^{\prime}(s) \exp \left(\frac{-\alpha}{1-\alpha}(s-t)\right) d s,
\end{aligned}
$$

left and right derivatives in the Riemann-Liouville sense

$$
{ }_{a}^{C F R} D^{\alpha} f(t)=\frac{M(\alpha)}{1-\alpha} \frac{d}{d t} \int_{a}^{t} f(s) \exp \left(\frac{-\alpha}{1-\alpha}(t-s)\right) d s,
$$

where $f \in H^{1}(a, b), a<b, \alpha \in[0,1]$,

$$
{ }^{C F R} D_{b}^{\alpha} f(t)=\frac{-M(\alpha)}{1-\alpha} \frac{d}{d t} \int_{t}^{b} f(s) \exp \left(\frac{-\alpha}{1-\alpha}(s-t)\right) d s,
$$

where $M(\alpha)>0$ is a normalization function with $M(0)=M(1)=1$.

Definition 2 ([1]) Left and right fractional integrals for fractional derivatives with exponential kernel are defined respectively by

$$
\begin{aligned}
& { }_{a}^{C F} I^{\alpha} f(t)=\frac{1-\alpha}{M(\alpha)} f(t)+\frac{\alpha}{M(\alpha)} \int_{a}^{t} f(s) d s, \\
& { }^{C F} I_{b}^{\alpha} f(t)=\frac{1-\alpha}{M(\alpha)} f(t)+\frac{\alpha}{M(\alpha)} \int_{t}^{b} f(s) d s .
\end{aligned}
$$

Theorem 3 ([25]) Let $\alpha>0, p \geq 1, q \geq 1, \frac{1}{p}+\frac{1}{q} \leq 1+\alpha, f(x) \in{ }^{C F} I_{b}^{\alpha}\left(L_{p}\right)$, and $g(x) \in$ ${ }_{a}^{C F} I^{\alpha}\left(L_{q}\right)$, then integration by parts formulas are given as follows:

$$
\int_{a}^{b} f(x){ }_{a}^{C F R} D^{\alpha} g(x) d x=\int_{a}^{b}{ }^{C F R} D_{b}^{\alpha} f(x) g(x) d x
$$




$$
\begin{aligned}
& \int_{a}^{b} g(t){ }_{a}^{C F C} D^{\alpha} f(t) d t=\int_{a}^{b}{ }^{C F R} D_{b}^{\alpha} g(t) f(t) d t+\left.\frac{B(\alpha)}{1-\alpha} f(t) e_{\frac{-\alpha}{1-\alpha}, b-} g(t)\right|_{a} ^{b}, \\
& \int_{a}^{b} g(t){ }^{C F C} D_{b}^{\alpha} f(t) d t=\int_{a}^{b}{ }^{C F R} D^{\alpha} g(t) f(t) d t-\left.\frac{B(\alpha)}{1-\alpha} f(t) e_{\frac{-\alpha}{1-\alpha}, a^{+}} g(t)\right|_{a} ^{b}
\end{aligned}
$$

where $e_{\frac{-\alpha}{1-\alpha}, b^{-}}$and $e_{\frac{-\alpha}{1-\alpha}, a^{+}}$are the left and right exponential integral operators respectively,

$$
\begin{aligned}
& e_{\frac{-\alpha}{1-\alpha}, b^{-}}=\int_{a}^{x} e^{\frac{-\alpha}{1-\alpha}(t-a)} \varphi(t) d t, \quad x>a, \\
& e_{\frac{-\alpha}{1-\alpha}, a^{+}}=\int_{x}^{b} e^{\frac{-\alpha}{1-\alpha}(b-t)} \varphi(t) d t, \quad x<b,
\end{aligned}
$$

and function spaces ${ }^{C F} I_{b}^{\alpha}\left(L_{p}\right)$ and ${ }_{a}^{C F} I^{\alpha}\left(L_{q}\right)$ are defined by

$$
\begin{aligned}
& { }_{a}^{C F} I^{\alpha}\left(L_{p}\right)=\left\{f: f={ }_{a}^{C F} I^{\alpha} \varphi, \varphi \in L_{p}(a, b)\right\}, \\
& { }^{C F} I_{b}^{\alpha}\left(L_{p}\right)=\left\{f: f={ }^{C F} I_{b}^{\alpha} \varphi, \varphi \in L_{p}(a, b)\right\} .
\end{aligned}
$$

Definition 4 ([2]) Fractional derivative with Mittag-Leffler kernel is defined as follows: left and right derivatives in the Caputo sense

$$
\begin{aligned}
& { }_{a}^{A B C} D^{\alpha} f(t)=\frac{B(\alpha)}{1-\alpha} \int_{a}^{t} f^{\prime}(s) E_{\alpha}\left(\frac{-\alpha}{1-\alpha}(t-s)^{\alpha}\right) d s, \\
& { }^{A B C} D_{b}^{\alpha} f(t)=\frac{-B(\alpha)}{1-\alpha} \int_{t}^{b} f^{\prime}(s) E_{\alpha}\left(\frac{-\alpha}{1-\alpha}(s-t)^{\alpha}\right) d s,
\end{aligned}
$$

left and right derivatives in the Riemann-Liouville sense

$$
{ }_{a}^{A B R} D^{\alpha} f(t)=\frac{B(\alpha)}{1-\alpha} \frac{d}{d t} \int_{a}^{t} f(s) E_{\alpha}\left(\frac{-\alpha}{1-\alpha}(t-s)^{\alpha}\right) d s
$$

where $f \in H^{1}(a, b), a<b, \alpha \in[0,1]$,

$$
{ }^{A B R} D_{b}^{\alpha} f(t)=\frac{-B(\alpha)}{1-\alpha} \frac{d}{d t} \int_{t}^{b} f(s) E_{\alpha}\left(\frac{-\alpha}{1-\alpha}(s-t)^{\alpha}\right) d s,
$$

where $B(\alpha)>0$ is a normalization function with $B(0)=B(1)=1$.

Definition 5 ([26]) Left and right fractional integrals for fractional derivative with MittagLeffler kernel are defined respectively by

$$
\begin{aligned}
& { }_{a}^{A B} I^{\alpha} f(t)=\frac{1-\alpha}{B(\alpha)} f(t)+\frac{\alpha}{B(\alpha)} I_{a}^{\alpha} f(t), \\
& { }^{A B} I_{b}^{\alpha} f(t)=\frac{1-\alpha}{B(\alpha)} f(t)+\frac{\alpha}{B(\alpha)} I_{b}^{\alpha} f(t) s .
\end{aligned}
$$


Theorem $6([1,8])$ The Laplace transform of fractional definitions with exponential kernel (1) and (3) is given as follows:

$$
\begin{aligned}
& \mathfrak{L}\left\{{ }_{a}^{C F R} D^{\alpha} f(t)\right\}(s)=\frac{M(\alpha)}{1-\alpha} \frac{s \mathfrak{L}\{f(t)\}(s)}{s+\frac{\alpha}{1-\alpha}}, \\
& \mathfrak{L}\left\{\left({ }_{a}^{C F C} D^{\alpha} f\right)(t)\right\}(s)=\frac{M(\alpha)}{1-\alpha} \frac{s \mathfrak{L}\{f(t)\}(s)}{s+\frac{\alpha}{1-\alpha}}-\frac{M(\alpha)}{1-\alpha} f(a) e^{-a s} \frac{1}{s+\frac{\alpha}{1-\alpha}} .
\end{aligned}
$$

Theorem 7 ([3]) The Laplace transform offractional definitions with Mittag-Leffler kernel (8) and (10) is given as follows:

$$
\begin{aligned}
& \mathfrak{L}\left\{{ }_{a}^{A B R} D^{\alpha} f(t)\right\}(s)=\frac{B(\alpha)}{1-\alpha} \frac{s^{\alpha} \mathfrak{L}\{f(t)\}(s)}{s^{\alpha}+\frac{\alpha}{1-\alpha}}, \\
& \mathfrak{L}\left\{{ }_{a}^{A B C} D^{\alpha} f(t)\right\}(s)=\frac{B(\alpha)}{1-\alpha} \frac{s^{\alpha} \mathfrak{L}\{f(t)\}(s)-s^{\alpha-1} f(a)}{s^{\alpha}+\frac{\alpha}{1-\alpha}} .
\end{aligned}
$$

Definition 8 The convolution of $f(t)$ and $g(t)$ is defined as follows:

$$
(f * g)(t)=\int_{0}^{t} f(s) g(t-s) d s, \quad f, g:[0, \infty) \rightarrow \mathrm{R} .
$$

Definition 9 ([41]) The Mittag-Leffler function $E_{\delta}(z)$ is defined by

$$
E_{\delta}(z)=\sum_{k=0}^{\infty} \frac{z^{k}}{\Gamma(\delta k+1)} \quad(z \in \mathrm{C}, \operatorname{Re}(\delta)>0),
$$

and the Mittag-Leffler function with two parameters is defined by

$$
E_{\delta, \theta}(z)=\sum_{k=0}^{\infty} \frac{z^{k}}{\Gamma(\delta k+\theta)} \quad(z, \theta \in \mathrm{C}, \operatorname{Re}(\delta)>0) .
$$

Property 10 The inverse Laplace transform of some special functions has the following properties:

(i) $\mathfrak{L}^{-1}\left\{\frac{a}{s\left(s^{\delta}+a\right)}\right\}=1-E_{\delta}\left(-a t^{\delta}\right)$,

(ii) $\mathfrak{L}^{-1}\left\{\frac{1}{s^{\delta}+a}\right\}=t^{\delta-1} E_{\delta, \delta}\left(-a t^{\delta}\right)$.

Property $11 \mathfrak{L}\{(f * g)(t)\}=\mathfrak{L}\{f(t)\} \mathfrak{L}\{g(t)\}$.

In the following section, firstly we give fractional S-L problems within different versions of the operators with exponential kernel in the Riemann-Liouville and Caputo sense and compare them. Secondly, we give fractional S-L problems within different versions of the operators with Mittag-Leffler kernel in the Riemann-Liouville and Caputo sense and compare them. We obtain the representation of solutions for S-L problems by the Laplace transform and find the analytical solutions of the problem. We analyze the solutions of these different versions and display them by simulation under different potentials, different orders, and different eigenvalues. Finally, we compare the solutions of the problem with these different versions, and we also compare the solutions of the problem with exponential and Mittag-Leffler kernels together by simulation. 


\section{Main results}

\subsection{Representations of solutions of fractional Sturm-Liouville problems with exponential and Mittag-Leffler kernels}

Theorem 12 Let us consider the fractional Sturm-Liouville initial value problem with exponential kernel:

$$
\begin{aligned}
& { }^{C F} L_{1} f={ }_{0}^{C F C} D^{\alpha}\left({ }_{0}^{C F R} D^{\alpha} f(x)\right)+q(x) f(x)=\lambda f(x), \quad x \in[0, n], n \in \mathrm{R}^{+}, \\
& { }_{0}^{C F R} D^{\alpha} f(0)=c_{1},
\end{aligned}
$$

where $0<\alpha<1, q(x)$ is a real-valued continuous function on $[0, n]$. Then the representation of solution of problem (12)-(13) is as follows: $\lambda \neq\{0,1\}$,

$$
\begin{aligned}
& f(x, \lambda)=c_{1} \frac{e^{\frac{\alpha \lambda x}{(\alpha-1)(\lambda-1)}}\left(\sinh \left(\frac{\alpha \sqrt{\lambda} x}{(\alpha-1)(\lambda-1)}\right)+\sqrt{\lambda} \cosh \left(\frac{\alpha \sqrt{\lambda} x}{(\alpha-1)(\lambda-1)}\right)\right)}{(\alpha-1)(\lambda-1) \sqrt{\lambda}} \\
& +\frac{\alpha\left[-2 \sqrt{\lambda}+(\lambda+1)+(\lambda+1)\left(-e^{\frac{2 \alpha \lambda x}{(\lambda-1)(\alpha-1) \sqrt{\lambda}}}\right)\right] e^{-\frac{x \alpha(\sqrt{\lambda}-\lambda)}{(\alpha-1)(\lambda-1)}}}{2(\alpha-1)(\lambda-1)^{2} \sqrt{\lambda}} \\
& +\frac{\alpha\left(-2 \sqrt{\lambda} e^{\frac{2 \alpha \sqrt{\lambda} x}{(\lambda-1)(\alpha-1)}}\right) e^{-\frac{x \alpha(\sqrt{\lambda}-\lambda)}{(\alpha-1)(\lambda-1)}}}{2(\alpha-1)(\lambda-1)^{2} \sqrt{\lambda}} \\
& +\left(\frac{\alpha\left[-2 \sqrt{\lambda}+(\lambda+1)+(\lambda+1)\left(-e^{\frac{2 \alpha \sqrt{\lambda} x}{(\alpha-1)(\lambda-1)}}\right)\right] e^{-\frac{x \alpha(\sqrt{\lambda}-\lambda)}{(\alpha-1)(\lambda-1)}}}{2(\alpha-1)(\lambda-1)^{2} \sqrt{\lambda}}\right) * q(x) f(x) \\
& +\left(\frac{\alpha\left(-2 \sqrt{\lambda} e^{\frac{2 \alpha \lambda x}{(\alpha-1)(\lambda-1) \sqrt{\lambda}}}\right) e^{\left(-\frac{x \alpha(\sqrt{\lambda}-\lambda)}{(\alpha-1)(\lambda-1)}\right)}}{2(\alpha-1)(\lambda-1)^{2} \sqrt{\lambda}}-\frac{\delta(x)}{\lambda-1}\right) * q(x) f(x) .
\end{aligned}
$$

Proof If we apply the Laplace transform to both sides of equation (12) and by the help of Theorem 6, let $q(x) f(x)=g(x)$,

$$
\begin{aligned}
& \mathfrak{L}\left\{{ }_{0}^{C F C} D^{\alpha}\left({ }_{0}^{C F R} D^{\alpha} f\right)\right\}(s)+\mathfrak{L}\{g\}(s)=\lambda \mathfrak{L}\{f\}(s) \\
& =\frac{M(\alpha)}{1-\alpha} \frac{s \mathfrak{L}\left\{\left({ }_{0}^{C F R} D^{\alpha} f\right)\right\}(s)}{s+\frac{\alpha}{1-\alpha}}-\frac{M(\alpha)}{1-\alpha} \frac{\left({ }_{0}^{C F R} D^{\alpha} f\right)(0)}{s+\frac{\alpha}{1-\alpha}}+\mathfrak{L}\{g\}(s)=\lambda \mathfrak{L}\{f\}(s) \\
& =\frac{M(\alpha)}{1-\alpha} \frac{s \frac{M(\alpha)}{1-\alpha} \frac{s \mathfrak{L}\{(f)\}(s)}{s+\frac{\alpha}{1-\alpha}}}{s+\frac{\alpha}{1-\alpha}}-\frac{M(\alpha)}{1-\alpha} \frac{\left({ }_{0}^{C F R} D^{\alpha} f\right)(0)}{s+\frac{\alpha}{1-\alpha}}+\mathfrak{L}\{g\}(s)=\lambda \mathfrak{L}\{f\}(s) \\
& =\left[\frac{M(\alpha)}{1-\alpha} \frac{s}{s+\frac{\alpha}{1-\alpha}}\right]^{2} \mathfrak{L}\{(f)\}(s)-c_{1} \frac{M(\alpha)}{1-\alpha} \frac{1}{s+\frac{\alpha}{1-\alpha}}+\mathfrak{L}\{g\}(s)=\lambda \mathfrak{L}\{f\}(s) \\
& \Rightarrow \quad \mathfrak{L}\{(f)\}(s)=\frac{c_{1} \frac{M(\alpha)}{1-\alpha} \frac{1}{s+\frac{\alpha}{1-\alpha}}}{\left(\frac{M(\alpha)}{1-\alpha} \frac{s}{s+\frac{\alpha}{1-\alpha}}\right)^{2}-\lambda}-\frac{1}{\left(\frac{M(\alpha)}{1-\alpha} \frac{s}{s+\frac{\alpha}{1-\alpha}}\right)^{2}-\lambda} \mathfrak{L}\{g\}(s) .
\end{aligned}
$$

Now, if we apply the inverse Laplace transform to equation (15) and use a convolution theorem, so we can find the sum representation of solution, noted by (14), of problem (12)-(13). 
Theorem 13 Let us consider the fractional Sturm-Liouville initial value problem with exponential kernel:

$$
\begin{aligned}
& { }^{C F} L_{2} f={ }_{0}^{C F C} D^{\alpha}\left({ }_{0}^{C F C} D^{\alpha} f(x)\right)+q(x) f(x)=\lambda f(x), \quad x \in[0, n], n \in \mathrm{R}^{+}, \\
& { }_{0}^{C F C} D^{\alpha} f(0)=c_{1}, \quad f(0)=c_{2},
\end{aligned}
$$

where $0<\alpha<1, q(x)$ is a real-valued continuous function on $[0, n]$. Then the representation of solution of problem (16)-(17) is as follows: $\lambda \neq\{0,1\}$,

$$
\begin{aligned}
f(x, \lambda)= & c_{1}\left(-\frac{-\alpha\left(e^{\frac{x \alpha \sqrt{\lambda}}{1+(\alpha-1) \sqrt{\lambda}}}(-1+(\alpha-1) \sqrt{\lambda})^{2}+e^{\frac{x \alpha \sqrt{\lambda}}{-1+(\alpha-1) \sqrt{\lambda}}}(1+(\alpha-1) \sqrt{\lambda})^{2}\right)}{2\left(-1+(\alpha-1)^{2} \lambda\right)^{2}}\right. \\
& \left.+\frac{(\alpha-1) \delta(x)}{-1+(\alpha-1)^{2} \lambda}\right) \\
& -c_{2} \frac{\left(e^{\frac{x \alpha \sqrt{\lambda}}{1+(\alpha-1) \sqrt{\lambda}}}(1-(\alpha-1) \sqrt{\lambda})+e^{\frac{x \alpha \sqrt{\lambda}}{-1+(\alpha-1) \sqrt{\lambda}}}(1+(\alpha-1) \sqrt{\lambda})\right)}{2\left(-1+(\alpha-1)^{2} \lambda\right)} \\
& +\left(\frac{-\alpha\left(-e^{\frac{x \alpha \sqrt{\lambda}}{1+(\alpha-1) \sqrt{\lambda}}}(-1+(\alpha-1) \sqrt{\lambda})^{2}+e^{\frac{x \alpha \sqrt{\lambda}}{-1+(\alpha-1) \sqrt{\lambda}}}(1+(\alpha-1) \sqrt{\lambda})^{2}\right)}{2 \sqrt{\lambda}\left(-1+(\alpha-1)^{2} \lambda\right)^{2}}\right. \\
& \left.-\frac{(\alpha-1)^{2} \delta(x)}{-1+(\alpha-1)^{2} \lambda}\right) * q(x) f(x) .
\end{aligned}
$$

Proof Proof is straightforward from the proof of Theorem 12.

Theorem 14 Let us consider the fractional Sturm-Liouville initial value problem with exponential kernel:

$$
\begin{aligned}
& { }^{C F} L_{3} f={ }_{0}^{C F R} D^{\alpha}\left({ }_{0}^{C F C} D^{\alpha} f(x)\right)+q(x) f(x)=\lambda f(x), \quad x \in[0, n], n \in \mathrm{R}^{+}, \\
& f(0)=c_{2},
\end{aligned}
$$

where $0<\alpha<1, q(x)$ is a real-valued continuous function on $[0, n]$. Then the representation of solution of problem (19)-(20) is as follows: $\lambda \neq\{0,1\}$,

$$
\begin{aligned}
f(x, \lambda)= & -c_{2}\left(-\frac{e^{\frac{x \alpha \sqrt{\lambda}}{1+(\alpha-1) \sqrt{\lambda}}}(1-(\alpha-1) \sqrt{\lambda})+e^{\frac{x \alpha \sqrt{\lambda}}{-1+(\alpha-1) \sqrt{\lambda}}}(1+(\alpha-1) \sqrt{\lambda})}{2(1-\alpha)\left(-1+(\alpha-1)^{2} \lambda\right)^{2}}\right) \\
& -\left(\frac{\alpha\left(-e^{\frac{x \alpha \sqrt{\lambda}}{1+(\alpha-1) \sqrt{\lambda}}}(-1+(\alpha-1) \sqrt{\lambda})^{2}+e^{\frac{x \alpha \sqrt{\lambda}}{-1+(\alpha-1) \sqrt{\lambda}}}(1+(\alpha-1) \sqrt{\lambda})^{2}\right)}{2 \sqrt{\lambda}\left(-1+(\alpha-1)^{2} \lambda\right)^{2}}\right. \\
& \left.-\frac{(\alpha-1)^{2} \delta(x)}{-1+(\alpha-1)^{2} \lambda}\right) * q(x) f(x) .
\end{aligned}
$$

Proof Proof is straightforward from the proof of Theorem 12.

Remark Approximate solutions of the problems according to the Mittag-Leffler function $E_{\delta, \theta}(z)=\sum_{k=0}^{500} \frac{z^{k}}{\Gamma(\delta k+\theta)}$ will be simulated in all of the figures, also let $B(\alpha)=M(\alpha)=1$. 
Theorem 15 Let us consider the fractional Sturm-Liouville initial value problem with exponential kernel:

$$
{ }^{C F} L_{4} f={ }_{0}^{C F R} D^{\alpha}\left({ }_{0}^{C F R} D^{\alpha} f(x)\right)+q(x) f(x)=\lambda f(x), \quad x \in[0, n],
$$

where $0<\alpha<1, q(x)$ is a real-valued continuous function on $[0, n]$. Then the representation of solution of equation (22) is as follows: $\lambda \neq\{0,1\}$,

$$
\begin{aligned}
f(x, \lambda)= & -\left(\frac{\alpha\left(-e^{\frac{x \alpha \sqrt{\lambda}}{1+(\alpha-1) \sqrt{\lambda}}}(-1+(\alpha-1) \sqrt{\lambda})^{2}+e^{\frac{x \alpha \sqrt{\lambda}}{-1+(\alpha-1) \sqrt{\lambda}}}(1+(\alpha-1) \sqrt{\lambda})^{2}\right)}{2 \sqrt{\lambda}\left(-1+(\alpha-1)^{2} \lambda\right)^{2}}\right. \\
& \left.-\frac{(\alpha-1)^{2} \delta(x)}{-1+(\alpha-1)^{2} \lambda}\right) * q(x) f(x) .
\end{aligned}
$$

Proof Proof is straightforward from the proof of Theorem 12.

Theorem 16 Let us consider the fractional Sturm-Liouville initial value problem with Mittag-Leffler kernel:

$$
\begin{aligned}
& { }^{A B} L_{1} f={ }_{0}^{A B C} D^{\alpha}\left({ }_{0}^{A B R} D^{\alpha} f(x)\right)+q(x) f(x)=\lambda f(x), \quad x \in[0,1], \\
& { }_{0}^{A B R} D^{\alpha} f(0)=c_{3},
\end{aligned}
$$

where $0<\alpha<1, q(x)$ is a real-valued continuous function on $[0,1]$. Then the representation of solution of problem (24)-(25) is as follows: $\lambda \neq\{0,1\}$,

$$
\begin{aligned}
f(x, \lambda)= & c_{3}\left[\frac{(1-\alpha)}{1-\lambda(1-\alpha)^{2}}-\frac{\sqrt{\lambda}(1-\alpha)^{2}}{B(\alpha)\left(B^{2}(\alpha)-\lambda(1-\alpha)^{2}\right)}\left\{1-E_{\alpha}\left(\frac{\alpha \sqrt{\lambda}}{B(\alpha)-\sqrt{\lambda}(1-\alpha)} x^{\alpha}\right)\right\}\right. \\
& +\frac{\sqrt{\lambda}(1-\alpha)^{2}}{B(\alpha)\left(B^{2}(\alpha)-\lambda(1-\alpha)^{2}\right)}\left\{1-E_{\alpha}\left(\frac{-\alpha \sqrt{\lambda}}{B(\alpha)+\sqrt{\lambda}(1-\alpha)} x^{\alpha}\right)\right\} \\
& -\frac{(1-\alpha)}{2 B(\alpha)(B(\alpha)+\sqrt{\lambda}(1-\alpha))}\left\{1-E_{\alpha}\left(\frac{\alpha \sqrt{\lambda}}{B(\alpha)-\sqrt{\lambda}(1-\alpha)} x^{\alpha}\right)\right\} \\
& -\frac{(1-\alpha)}{2 B(\alpha)(B(\alpha)-\sqrt{\lambda}(1-\alpha))}\left\{1-E_{\alpha}\left(\frac{-\alpha \sqrt{\lambda}}{B(\alpha)+\sqrt{\lambda}(1-\alpha)} x^{\alpha}\right)\right\} \\
& +\alpha\left(\frac{1}{-2 B(\alpha) \sqrt{\lambda}}\left\{1-E_{\alpha}\left(\frac{\alpha \sqrt{\lambda}}{B(\alpha)-\sqrt{\lambda}(1-\alpha)} x^{\alpha}\right)\right\}\right. \\
& \left.\left.+\frac{1}{2 B(\alpha) \sqrt{\lambda}}\left\{1-E_{\alpha}\left(\frac{-\alpha \sqrt{\lambda}}{B(\alpha)+\sqrt{\lambda}(1-\alpha)} x^{\alpha}\right)\right\}\right)\right] \\
& +\left[\frac{\delta(x)}{B^{2}(\alpha)-\lambda(1-\alpha)^{2}}\right. \\
& +\frac{\lambda \alpha(1-\alpha)}{B(\alpha)\left(B^{2}(\alpha)-\lambda(1-\alpha)^{2}\right)}\left[\frac{x^{\alpha-1}}{B(\alpha)-\sqrt{\lambda}(1-\alpha)} E_{\alpha, \alpha}\left(\frac{\alpha \sqrt{\lambda}}{B(\alpha)-\sqrt{\lambda}(1-\alpha)} x^{\alpha}\right)\right. \\
& \left.+\frac{x^{\alpha-1}}{B(\alpha)+\sqrt{\lambda}(1-\alpha)} E_{\alpha, \alpha}\left(\frac{-\alpha \sqrt{\lambda}}{B(\alpha)+\sqrt{\lambda}(1-\alpha)} x^{\alpha}\right)\right] \\
& +\frac{\sqrt{\lambda} \alpha}{B^{2}(\alpha)-\lambda(1-\alpha)^{2}}\left[\frac{x^{\alpha-1}}{2 B(\alpha)} E_{\alpha, \alpha}\left(\frac{\alpha \sqrt{\lambda}}{B(\alpha)-\sqrt{\lambda}(1-\alpha)} x^{\alpha}\right)\right.
\end{aligned}
$$




$$
\begin{aligned}
& \left.-\frac{x^{\alpha-1}}{2 B(\alpha)} E_{\alpha, \alpha}\left(\frac{-\alpha \sqrt{\lambda}}{B(\alpha)+\sqrt{\lambda}(1-\alpha)} x^{\alpha}\right)\right] \\
& +\frac{\alpha(1-\alpha)}{B(\alpha)}\left[\frac{x^{\alpha-1}}{B(\alpha)-\sqrt{\lambda}(1-\alpha)} E_{\alpha, \alpha}\left(\frac{\alpha \sqrt{\lambda}}{B(\alpha)-\sqrt{\lambda}(1-\alpha)} x^{\alpha}\right)\right. \\
& \left.+\frac{x^{\alpha-1}}{B(\alpha)+\sqrt{\lambda}(1-\alpha)} E_{\alpha, \alpha}\left(\frac{-\alpha \sqrt{\lambda}}{B(\alpha)+\sqrt{\lambda}(1-\alpha)} x^{\alpha}\right)\right] \\
& +\alpha\left(\frac{x^{\alpha-1}}{2 \sqrt{\lambda} B(\alpha)} E_{\alpha, \alpha}\left(\frac{\alpha \sqrt{\lambda}}{B(\alpha)-\sqrt{\lambda}(1-\alpha)} x^{\alpha}\right)\right. \\
& \left.\left.-\frac{x^{\alpha-1}}{2 \sqrt{\lambda} B(\alpha)} E_{\alpha, \alpha}\left(\frac{-\alpha \sqrt{\lambda}}{B(\alpha)+\sqrt{\lambda}(1-\alpha)} x^{\alpha}\right)\right)\right] * q(x) f(x) .
\end{aligned}
$$

Proof Let us apply the Laplace transform to both sides of equation (24) and, by the help of Theorem 7, let $q(x) f(x)=g(x)$,

$$
\begin{aligned}
& \mathfrak{L}\left\{{ }_{0}^{A B C} D^{\alpha}\left({ }_{0}^{A B R} D^{\alpha} f\right)\right\}(s)+\mathfrak{L}\{g\}(s)=\lambda \mathfrak{L}\{f\}(s) \\
&=\frac{B(\alpha)}{1-\alpha} \frac{s^{\alpha} \mathfrak{L}\left\{\left({ }_{0}^{A B R} D^{\alpha} f\right)\right\}(s)}{s^{\alpha}+\frac{\alpha}{1-\alpha}}-\frac{B(\alpha)}{1-\alpha} \frac{s^{\alpha-1}\left({ }_{0}^{A B R} D^{\alpha} f\right)(0)}{s^{\alpha}+\frac{\alpha}{1-\alpha}}+\mathfrak{L}\{g\}(s)=\lambda \mathfrak{L}\{f\}(s) \\
&=\frac{B(\alpha)}{1-\alpha} \frac{s \frac{B(\alpha)}{1-\alpha} \frac{s^{\alpha} \mathfrak{L}\{(f)\}(s)}{s^{\alpha}+\frac{\alpha}{1-\alpha}}}{s+\frac{\alpha}{1-\alpha}}-\frac{B(\alpha)}{1-\alpha} \frac{s^{\alpha-1}\left({ }_{0}^{A B R} D^{\alpha} f\right)(0)}{s^{\alpha}+\frac{\alpha}{1-\alpha}}+\mathfrak{L}\{g\}(s)=\lambda \mathfrak{L}\{f\}(s) \\
&=\left(\frac{B(\alpha)}{1-\alpha} \frac{s^{\alpha}}{s^{\alpha}+\frac{\alpha}{1-\alpha}}\right)^{2} \mathfrak{L}\{(f)\}(s)-c_{3} \frac{B(\alpha)}{1-\alpha} \frac{s^{\alpha-1}\left({ }_{0}^{A B R} D^{\alpha} f\right)(0)}{s^{\alpha}+\frac{\alpha}{1-\alpha}}+\mathfrak{L}\{g\}(s)=\lambda \mathfrak{L}\{f\}(s) \\
& \Rightarrow \quad \mathfrak{L}\{(f)\}(s)=c_{1} \frac{s^{\alpha-1}}{\frac{B(\alpha)}{1-\alpha}\left(\frac{s^{2 \alpha}}{s^{\alpha}+\frac{\alpha}{1-\alpha}}\right)-\lambda\left(s^{\alpha}+\frac{\alpha}{1-\alpha}\right)}-\frac{1}{\left(\frac{B(\alpha)}{1-\alpha} \frac{s^{\alpha}}{s^{\alpha}+\frac{\alpha}{1-\alpha}}\right)^{2}-\lambda} \mathfrak{L}\{g\}(s) .
\end{aligned}
$$

Now, if we apply the inverse Laplace transform to the last equation and use a convolution theorem, so we can find the sum representation of solution, noted by (26), of problem (24) $-(25)$.

Theorem 17 Let us consider the fractional Sturm-Liouville initial value problem with Mittag-Leffler kernel:

$$
\begin{aligned}
& { }^{A B} L_{2} f={ }_{0}^{A B C} D^{\alpha}\left({ }_{0}^{A B C} D^{\alpha} f(x)\right)+q(x) f(x)=\lambda f(x), \quad x \in[0,1], \\
& { }_{0}^{A B R} D^{\alpha} f(0)=c_{3}, \quad f(0)=c_{4},
\end{aligned}
$$

where $0<\alpha<1, q(x)$ is a real-valued continuous function on $[0, n]$. Then the representation of solution of problem (27)-(28) is as follows: $\lambda \neq\{0,1\}$,

$$
\begin{aligned}
f(x, \lambda)= & c_{3}\left[\frac{1}{B^{2}(\alpha)-\lambda(1-\alpha)^{2}}\right. \\
& +\frac{\sqrt{\lambda}(1-\alpha)}{B(\alpha)\left(B^{2}(\alpha)-\lambda(1-\alpha)^{2}\right)}\left\{E_{\alpha}\left(\frac{\alpha \sqrt{\lambda}}{B(\alpha)-\sqrt{\lambda}(1-\alpha)} x^{\alpha}\right)\right. \\
& \left.-E_{\alpha}\left(\frac{-\alpha \sqrt{\lambda}}{B(\alpha)+\sqrt{\lambda}(1-\alpha)} x^{\alpha}\right)\right\}
\end{aligned}
$$




$$
\begin{aligned}
& +\frac{1}{B(\alpha)(B(\alpha)+\sqrt{\lambda}(1-\alpha))}\left\{-\frac{1}{2}\left(1-E_{\alpha}\left(\frac{\alpha \sqrt{\lambda}}{B(\alpha)-\sqrt{\lambda}(1-\alpha)} x^{\alpha}\right)\right)\right\} \\
& -\frac{1}{B(\alpha)(B(\alpha)+\sqrt{\lambda}(1-\alpha))}\left\{-\frac{1}{2}\left(1-E_{\alpha}\left(\frac{-\alpha \sqrt{\lambda}}{B(\alpha)+\sqrt{\lambda}(1-\alpha)} x^{\alpha}\right)\right)\right\} \\
& +\frac{(1-\alpha)}{\sqrt{\lambda} B(\alpha)}\left\{E_{\alpha}\left(\frac{\alpha \sqrt{\lambda}}{B(\alpha)-\sqrt{\lambda}(1-\alpha)} x^{\alpha}\right)\right. \\
& \left.-E_{\alpha}\left(\frac{-\alpha \sqrt{\lambda}}{B(\alpha)+\sqrt{\lambda}(1-\alpha)} x^{\alpha}\right)\right\} \\
& +\frac{1}{\lambda B(\alpha)}\left\{\frac{B(\alpha)-\sqrt{\lambda}(1-\alpha)}{-2}\left[1-E_{\alpha}\left(\frac{\alpha \sqrt{\lambda}}{B(\alpha)-\sqrt{\lambda}(1-\alpha)} x^{\alpha}\right)\right]\right. \\
& \left.-\frac{B(\alpha)+\sqrt{\lambda}(1-\alpha)}{2}\left[1-E_{\alpha}\left(\frac{-\alpha \sqrt{\lambda}}{B(\alpha)+\sqrt{\lambda}(1-\alpha)} x^{\alpha}\right)\right]\right\} \\
& +c_{4}\left[\frac{(1-\alpha)}{1-\lambda(1-\alpha)^{2}}-\frac{\sqrt{\lambda}(1-\alpha)^{2}}{B(\alpha)\left(B^{2}(\alpha)-\lambda(1-\alpha)^{2}\right)}\right. \\
& \times\left[1-E_{\alpha}\left(\frac{\alpha \sqrt{\lambda}}{B(\alpha)-\sqrt{\lambda}(1-\alpha)} x^{\alpha}\right)\right] \\
& +\frac{\sqrt{\lambda}(1-\alpha)^{2}}{B(\alpha)\left(B^{2}(\alpha)-\lambda(1-\alpha)^{2}\right)}\left[1-E_{\alpha}\left(\frac{-\alpha \sqrt{\lambda}}{B(\alpha)+\sqrt{\lambda}(1-\alpha)} x^{\alpha}\right)\right] \\
& -\frac{(1-\alpha)}{2 B(\alpha)(B(\alpha)+\sqrt{\lambda}(1-\alpha))}\left[1-E_{\alpha}\left(\frac{\alpha \sqrt{\lambda}}{B(\alpha)-\sqrt{\lambda}(1-\alpha)} x^{\alpha}\right)\right] \\
& -\frac{(1-\alpha)}{2 B(\alpha)(B(\alpha)-\sqrt{\lambda}(1-\alpha))}\left[1-E_{\alpha}\left(\frac{-\alpha \sqrt{\lambda}}{B(\alpha)+\sqrt{\lambda}(1-\alpha)} x^{\alpha}\right)\right] \\
& +\alpha\left(\frac{1}{-2 B(\alpha) \sqrt{\lambda}}\left\{1-E_{\alpha}\left(\frac{\alpha \sqrt{\lambda}}{B(\alpha)-\sqrt{\lambda}(1-\alpha)} x^{\alpha}\right)\right\}\right. \\
& \left.\left.+\frac{1}{2 B(\alpha) \sqrt{\lambda}}\left\{1-E_{\alpha}\left(\frac{-\alpha \sqrt{\lambda}}{B(\alpha)+\sqrt{\lambda}(1-\alpha)} x^{\alpha}\right)\right\}\right)\right] \\
& -\left[\frac{\delta(x)}{B^{2}(\alpha)-\lambda(1-\alpha)^{2}}\right. \\
& +\frac{\lambda \alpha(1-\alpha)}{B(\alpha)\left(B^{2}(\alpha)-\lambda(1-\alpha)^{2}\right)}\left[\frac{x^{\alpha-1}}{B(\alpha)-\sqrt{\lambda}(1-\alpha)} E_{\alpha, \alpha}\left(\frac{\alpha \sqrt{\lambda}}{B(\alpha)-\sqrt{\lambda}(1-\alpha)} x^{\alpha}\right)\right. \\
& \left.+\frac{x^{\alpha-1}}{B(\alpha)+\sqrt{\lambda}(1-\alpha)} E_{\alpha, \alpha}\left(\frac{-\alpha \sqrt{\lambda}}{B(\alpha)+\sqrt{\lambda}(1-\alpha)} x^{\alpha}\right)\right] \\
& +\frac{\lambda \alpha^{2}}{B^{2}(\alpha)-\lambda(1-\alpha)^{2}}\left[\frac{x^{\alpha-1}}{2 \alpha \sqrt{\lambda} B(\alpha)} E_{\alpha, \alpha}\left(\frac{\alpha \sqrt{\lambda}}{B(\alpha)-\sqrt{\lambda}(1-\alpha)} x^{\alpha}\right)\right. \\
& \left.-\frac{x^{\alpha-1}}{2 \alpha \sqrt{\lambda} B(\alpha)} E_{\alpha, \alpha}\left(\frac{-\alpha \sqrt{\lambda}}{B(\alpha)+\sqrt{\lambda}(1-\alpha)} x^{\alpha}\right)\right] \\
& +\frac{\alpha(1-\alpha)}{B(\alpha)}\left[\frac{x^{\alpha-1}}{B(\alpha)-\sqrt{\lambda}(1-\alpha)} E_{\alpha, \alpha}\left(\frac{\alpha \sqrt{\lambda}}{B(\alpha)-\sqrt{\lambda}(1-\alpha)} x^{\alpha}\right)\right. \\
& \left.+\frac{x^{\alpha-1}}{B(\alpha)-\sqrt{\lambda}(1-\alpha)} E_{\alpha, \alpha}\left(\frac{-\alpha \sqrt{\lambda}}{B(\alpha)+\sqrt{\lambda}(1-\alpha)} x^{\alpha}\right)\right]
\end{aligned}
$$




$$
\begin{aligned}
& +\alpha\left(\frac{x^{\alpha-1}}{2 \sqrt{\lambda} B(\alpha)} E_{\alpha, \alpha}\left(\frac{\alpha \sqrt{\lambda}}{B(\alpha)-\sqrt{\lambda}(1-\alpha)} x^{\alpha}\right)\right. \\
& \left.\left.-\frac{x^{\alpha-1}}{2 \sqrt{\lambda} B(\alpha)} E_{\alpha, \alpha}\left(\frac{-\alpha \sqrt{\lambda}}{B(\alpha)+\sqrt{\lambda}(1-\alpha)} x^{\alpha}\right)\right)\right] * q(x) f(x) .
\end{aligned}
$$

Proof Proof is straightforward from the proof of Theorem 16.

Theorem 18 Let us consider the fractional Sturm-Liouville initial value problem with Mittag-Leffler kernel:

$$
\begin{aligned}
& { }^{A B} L_{3} f={ }_{0}^{A B R} D^{\alpha}\left({ }_{0}^{A B C} D^{\alpha} f(x)\right)+q(x) f(x)=\lambda f(x), \quad x \in[0,1], \\
& f(0)=c_{4},
\end{aligned}
$$

where $0<\alpha<1, q(x)$ is a real-valued continuous function on $[0,1]$. Then the representation of solution of problem (30)-(31) is as follows: $\lambda \neq\{0,1\}$,

$$
\begin{aligned}
& f(x, \lambda)=c_{4}\left[\frac{1}{B^{2}(\alpha)-\lambda(1-\alpha)^{2}}\right. \\
& +\frac{\sqrt{\lambda}(1-\alpha)}{B(\alpha)\left(B^{2}(\alpha)-\lambda(1-\alpha)^{2}\right)}\left\{E_{\alpha}\left(\frac{\alpha \sqrt{\lambda}}{B(\alpha)-\sqrt{\lambda}(1-\alpha)} x^{\alpha}\right)\right. \\
& \left.-E_{\alpha}\left(\frac{-\alpha \sqrt{\lambda}}{B(\alpha)+\sqrt{\lambda}(1-\alpha)} x^{\alpha}\right)\right\} \\
& +\frac{1}{B(\alpha)(B(\alpha)+\sqrt{\lambda}(1-\alpha))}\left\{-\frac{1}{2}\left(1-E_{\alpha}\left(\frac{\alpha \sqrt{\lambda}}{B(\alpha)-\sqrt{\lambda}(1-\alpha)} x^{\alpha}\right)\right)\right\} \\
& -\frac{1}{B(\alpha)(B(\alpha)-\sqrt{\lambda}(1-\alpha))}\left\{-\frac{1}{2}\left(1-E_{\alpha}\left(\frac{-\alpha \sqrt{\lambda}}{B(\alpha)+\sqrt{\lambda}(1-\alpha)} x^{\alpha}\right)\right)\right\} \\
& +\frac{(1-\alpha)}{\sqrt{\lambda} B(\alpha)}\left\{E_{\alpha}\left(\frac{\alpha \sqrt{\lambda}}{B(\alpha)-\sqrt{\lambda}(1-\alpha)} x^{\alpha}\right)-E_{\alpha}\left(\frac{-\alpha \sqrt{\lambda}}{B(\alpha)+\sqrt{\lambda}(1-\alpha)} x^{\alpha}\right)\right\} \\
& +\frac{1}{\lambda B(\alpha)}\left\{\frac{B(\alpha)-\sqrt{\lambda}(1-\alpha)}{-2}\left[1-E_{\alpha}\left(\frac{\alpha \sqrt{\lambda}}{B(\alpha)-\sqrt{\lambda}(1-\alpha)} x^{\alpha}\right)\right]\right. \\
& \left.\left.-\frac{B(\alpha)+\sqrt{\lambda}(1-\alpha)}{2}\left[1-E_{\alpha}\left(\frac{-\alpha \sqrt{\lambda}}{B(\alpha)+\sqrt{\lambda}(1-\alpha)} x^{\alpha}\right)\right]\right\}\right] \\
& -\left[\frac{\delta(x)}{B^{2}(\alpha)-\lambda(1-\alpha)^{2}}\right. \\
& +\frac{\lambda \alpha(1-\alpha)}{B(\alpha)\left(B^{2}(\alpha)-\lambda(1-\alpha)^{2}\right)}\left[\frac{x^{\alpha-1}}{B(\alpha)-\sqrt{\lambda}(1-\alpha)} E_{\alpha, \alpha}\left(\frac{\alpha \sqrt{\lambda}}{B(\alpha)-\sqrt{\lambda}(1-\alpha)} x^{\alpha}\right)\right. \\
& \left.+\frac{x^{\alpha-1}}{B(\alpha)+\sqrt{\lambda}(1-\alpha)} E_{\alpha, \alpha}\left(\frac{-\alpha \sqrt{\lambda}}{B(\alpha)+\sqrt{\lambda}(1-\alpha)} x^{\alpha}\right)\right] \\
& +\frac{\lambda \alpha^{2}}{B^{2}(\alpha)-\lambda(1-\alpha)^{2}}\left[\frac{x^{\alpha-1}}{2 \alpha \sqrt{\lambda} B(\alpha)} E_{\alpha, \alpha}\left(\frac{\alpha \sqrt{\lambda}}{B(\alpha)-\sqrt{\lambda}(1-\alpha)} x^{\alpha}\right)\right. \\
& \left.-\frac{x^{\alpha-1}}{2 \alpha \sqrt{\lambda} B(\alpha)} E_{\alpha, \alpha}\left(\frac{-\alpha \sqrt{\lambda}}{B(\alpha)+\sqrt{\lambda}(1-\alpha)} x^{\alpha}\right)\right]
\end{aligned}
$$




$$
\begin{aligned}
& +\frac{\alpha(1-\alpha)}{B(\alpha)}\left[\frac{x^{\alpha-1}}{B(\alpha)-\sqrt{\lambda}(1-\alpha)} E_{\alpha, \alpha}\left(\frac{\alpha \sqrt{\lambda}}{B(\alpha)-\sqrt{\lambda}(1-\alpha)} x^{\alpha}\right)\right. \\
& \left.+\frac{x^{\alpha-1}}{B(\alpha)+\sqrt{\lambda}(1-\alpha)} E_{\alpha, \alpha}\left(\frac{-\alpha \sqrt{\lambda}}{B(\alpha)+\sqrt{\lambda}(1-\alpha)} x^{\alpha}\right)\right] \\
& +\alpha\left(\frac{x^{\alpha-1}}{2 \sqrt{\lambda} B(\alpha)} E_{\alpha, \alpha}\left(\frac{\alpha \sqrt{\lambda}}{B(\alpha)-\sqrt{\lambda}(1-\alpha)} x^{\alpha}\right)\right. \\
& \left.\left.-\frac{x^{\alpha-1}}{2 \sqrt{\lambda} B(\alpha)} E_{\alpha, \alpha}\left(\frac{-\alpha \sqrt{\lambda}}{B(\alpha)+\sqrt{\lambda}(1-\alpha)} x^{\alpha}\right)\right)\right] * q(x) f(x) .
\end{aligned}
$$

Proof Proof is straightforward from the proof of Theorem 16.

Theorem 19 Let us consider the fractional Sturm-Liouville initial value problem with Mittag-Leffler kernel:

$$
{ }^{A B} L_{4} f={ }_{0}^{A B R} D^{\alpha}\left({ }_{0}^{A B R} D^{\alpha} f(x)\right)+q(x) f(x)=\lambda f(x), \quad x \in[0,1],
$$

where $0<\alpha<1, q(x)$ is a real-valued continuous function on $[0,1]$. Then the representation of solution of equation (33) is as follows: $\lambda \neq\{0,1\}$,

$$
\begin{aligned}
f(x, \lambda)= & \frac{\delta(x)}{B^{2}(\alpha)-\lambda(1-\alpha)^{2}} \\
& +\frac{\lambda \alpha(1-\alpha)}{B(\alpha)\left(B^{2}(\alpha)-\lambda(1-\alpha)^{2}\right)}\left[\frac{x^{\alpha-1}}{B(\alpha)-\sqrt{\lambda}(1-\alpha)} E_{\alpha, \alpha}\left(\frac{\alpha \sqrt{\lambda}}{B(\alpha)-\sqrt{\lambda}(1-\alpha)} x^{\alpha}\right)\right. \\
& \left.+\frac{x^{\alpha-1}}{B(\alpha)+\sqrt{\lambda}(1-\alpha)} E_{\alpha, \alpha}\left(\frac{-\alpha \sqrt{\lambda}}{B(\alpha)+\sqrt{\lambda}(1-\alpha)} x^{\alpha}\right)\right] \\
& +\frac{\sqrt{\lambda} \alpha}{B^{2}(\alpha)-\lambda(1-\alpha)^{2}}\left[\frac{x^{\alpha-1}}{2 B(\alpha)} E_{\alpha, \alpha}\left(\frac{\alpha \sqrt{\lambda}}{B(\alpha)-\sqrt{\lambda}(1-\alpha)} x^{\alpha}\right)\right. \\
& \left.-\frac{x^{\alpha-1}}{2 B(\alpha)} E_{\alpha, \alpha}\left(\frac{-\alpha \sqrt{\lambda}}{B(\alpha)+\sqrt{\lambda}(1-\alpha)} x^{\alpha}\right)\right] \\
& +\frac{\alpha(1-\alpha)}{B(\alpha)}\left[\frac{x^{\alpha-1}}{B(\alpha)-\sqrt{\lambda}(1-\alpha)} E_{\alpha, \alpha}\left(\frac{\alpha \sqrt{\lambda}}{B(\alpha)-\sqrt{\lambda}(1-\alpha)} x^{\alpha}\right)\right. \\
& \left.+\frac{x^{\alpha-1}}{B(\alpha)+\sqrt{\lambda}(1-\alpha)} E_{\alpha, \alpha}\left(\frac{-\alpha \sqrt{\lambda}}{B(\alpha)+\sqrt{\lambda}(1-\alpha)} x^{\alpha}\right)\right] \\
& +\alpha\left[\frac{x^{\alpha-1}}{2 \sqrt{\lambda} B(\alpha)} E_{\alpha, \alpha}\left(\frac{\alpha \sqrt{\lambda}}{B(\alpha)-\sqrt{\lambda}(1-\alpha)} x^{\alpha}\right)\right. \\
& \left.-\frac{x^{\alpha-1}}{2 \sqrt{\lambda} B(\alpha)} E_{\alpha, \alpha}\left(\frac{-\alpha \sqrt{\lambda}}{B(\alpha)+\sqrt{\lambda}(1-\alpha)} x^{\alpha}\right)\right] * q(x) f(x)
\end{aligned}
$$

Proof Proof is straightforward from the proof of Theorem 16.

\section{Conclusion}

In this study, we have considered fractional Sturm-Liouville (S-L) problems within nonsingular operators. A fractional S-L problem with exponential and Mittag-Leffler kernels 
is given with different versions in the Riemann-Liouville and Caputo sense. Also, we obtain the representation of solutions for S-L problems by the Laplace transform and find analytical solutions of the problems. We analyze solutions of these different versions and display them by simulation under different potentials, different orders, and different eigenvalues. However, we compare the solutions of the problem with these different versions, and we also compare the solutions of the problem with exponential and Mittag-Leffler kernels together by simulation.

We compare the eigenfunctions of problems (12)-(13), (19)-(20), and (16)-(17) under different orders, and we observe that the eigenfunctions of the problems converge to each other as $x$ increases in Fig. 1, Fig. 2, and Fig. 3. We compare the eigenfunctions of problems (12)-(13), (19)-(20), and (16)-(17) with each other, and we observe that the eigenfunctions of the problems converge to each other as $x$ increases in Fig. 4 . We compare the eigenfunctions of problem (12)-(13) with different orders, different domains, different potential functions, and different eigenvalues in Fig. 5, Fig. 6, Fig. 7, and Fig. 8.
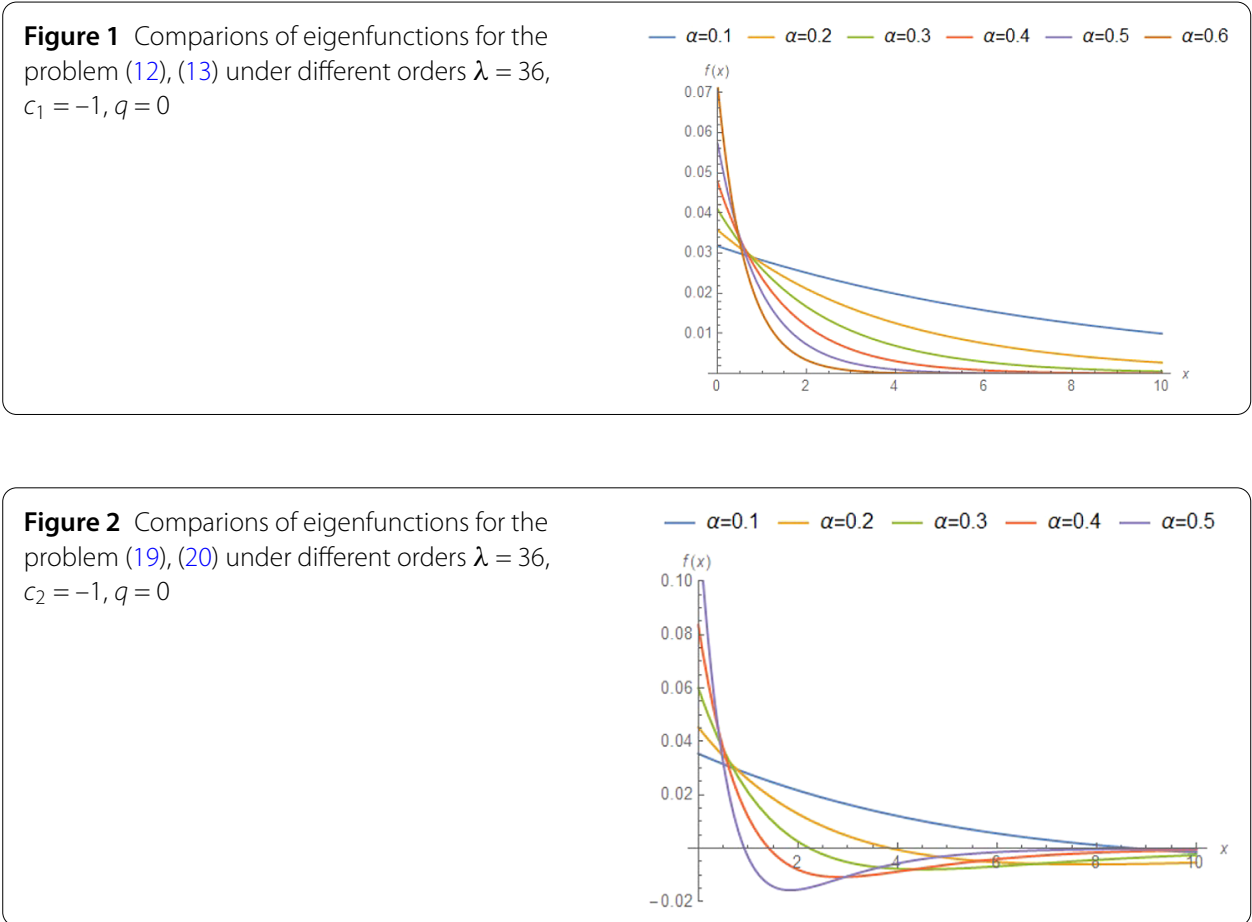

Figure 3 Comparions of eigenfunctions for the problem (16), (17) under different orders $\lambda=36$, $c_{1}=c_{2}=-1, q=0$

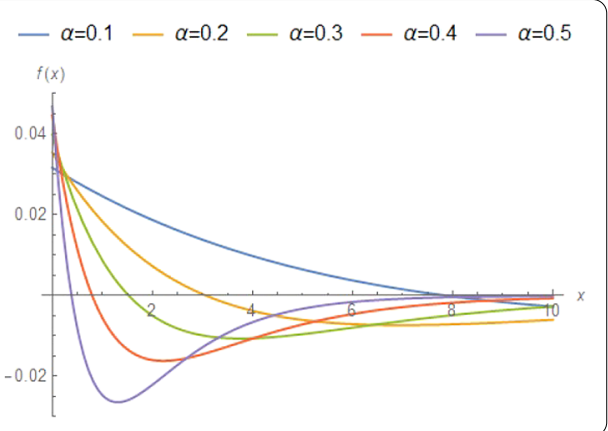


Figure 4 Comparions of eigenfunctions for the problems (19), (20)-(12), (13)-(16), (17) $\lambda=36$, $c_{1}=c_{2}=-1, q=0, \alpha=0.4$

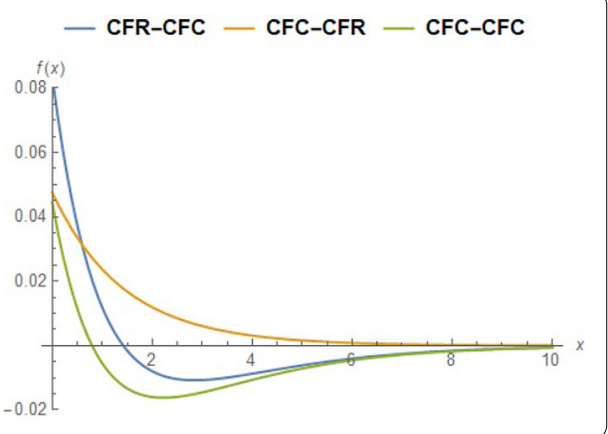

Figure 5 Comparions of eigenfunctions for the problem (12), (13) under different orders $c_{1}=-1$, $q=0, x=1$

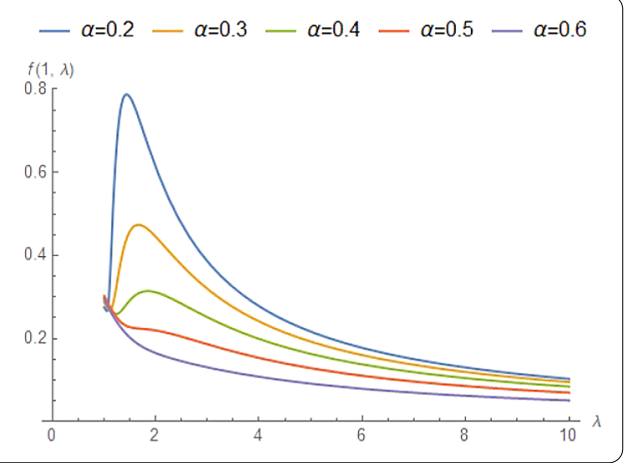

Figure 6 Comparions of eigenfunctions for the problem (12), (13) under different domains $c_{1}=-1$, $\alpha=0.4, q=0$

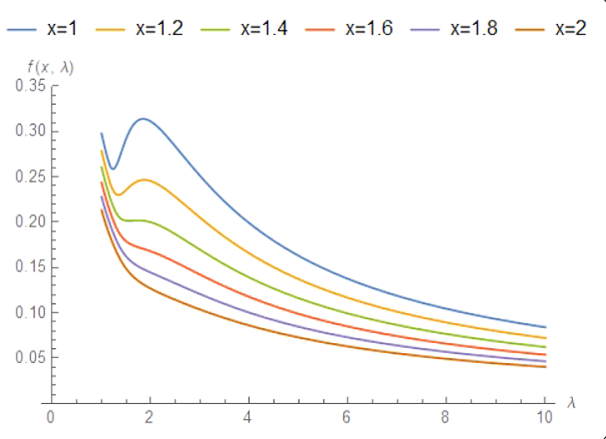

Figure 7 Comparions of eigenfunctions for the

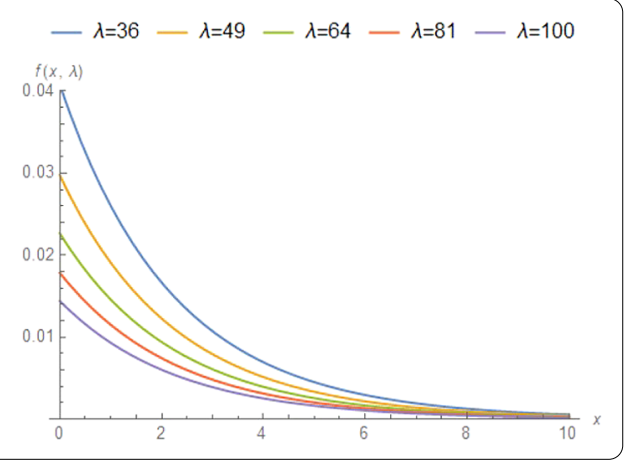
$c_{1}=-1, \alpha=0.3, q=0$

We compare the eigenfunctions of problems (24)-(25), (30)-(31), and (27)-(28) under different orders, and we observe that the eigenfunctions of the problems converge to each other as $x$ increases in Fig. 9, Fig. 10, and Fig. 11. We compare the eigenfunctions of prob- 
Figure 8 Comparions of eigenfunctions for the problem (12), (13) under different potentials $c_{1}=-1$, $\lambda=36, \alpha=0.3$ $-\mathrm{q}=0-\mathrm{q}=5-\mathrm{q}=10-\mathrm{q}=15$

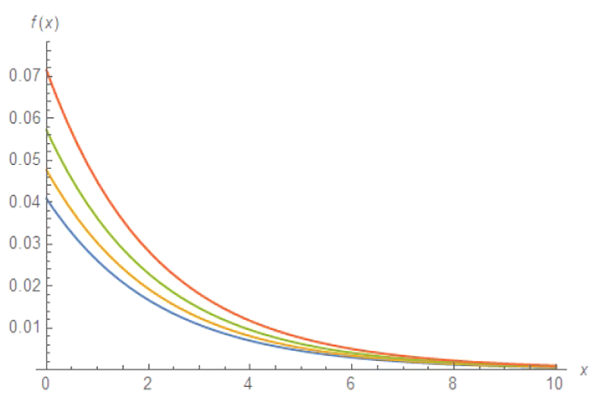

Figure 9 Comparions of eigenfunctions for the problem (24), (25) under different orders $\lambda=36$, $c_{3}=-1, q=0$

$-\alpha=0.1-\alpha=0.2-\alpha=0.3-\alpha=0.4-0.5$
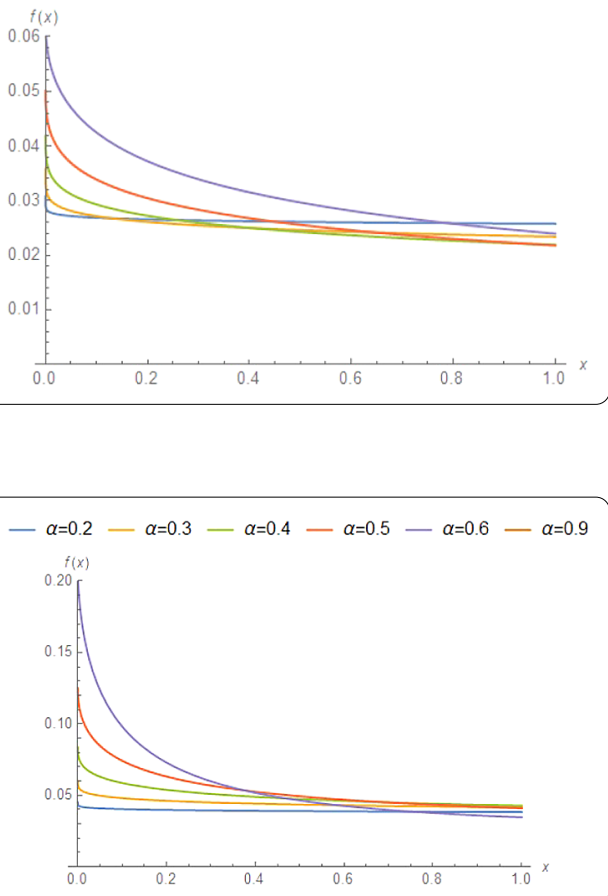

Figure 10 Comparions of eigenfunctions for the problem (30), (31) under different orders $\lambda=36$, $c_{4}=-1, q=0$

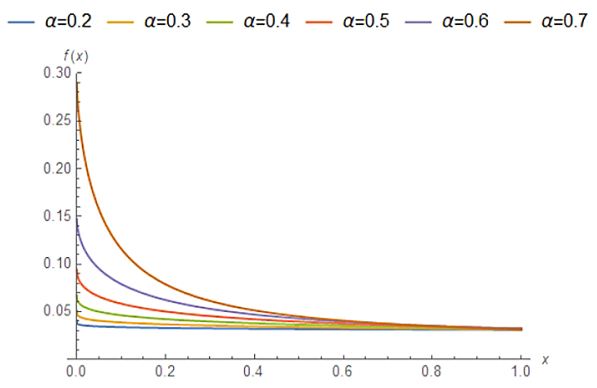

Figure 11 Comparions of eigenfunctions for the problem (27), (28) under different orders $\lambda=36$, $c_{3}=c_{4}=-1, q=0$

lems (24)-(25), (30)-(31), and (27)-(28) with each other in Fig. 12. We compare the eigenfunctions of problem (24)-(25) with different orders, different eigenvalues, different domains, and different potential functions in Fig. 13, Fig. 14, Fig. 15, and Fig. 16. 
Figure 12 Comparions of eigenfunctions for the problem (30), (31)-(24), (25)-(27), (28) $\lambda=36$, $\alpha=0.4, c_{3}=c_{4}=-1, q=0$
$-A B C-A B R-A B R-A B C-A B C-A B C$

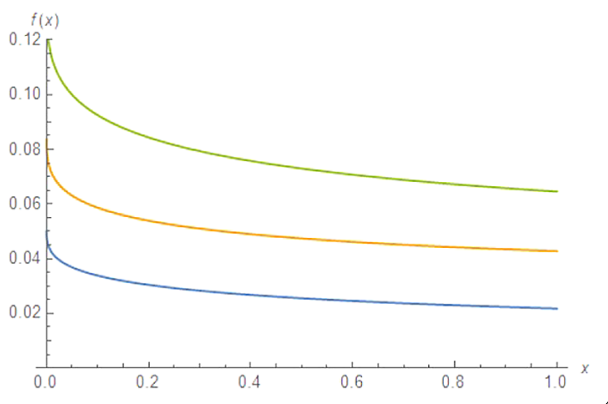

$-\alpha=0.1-\alpha=0.2-\alpha=0.3-\alpha=0.4-\alpha=0.5$

Figure 13 Comparions of eigenfunctions for the problem (24), (25) under different orders $c_{3}=1, q=0$, $x=0.5$

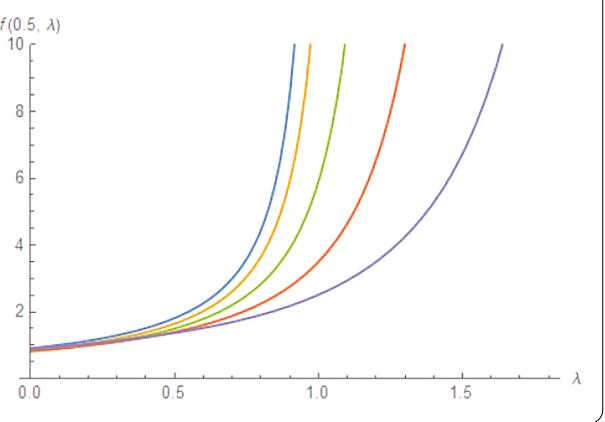

Figure 14 Comparions of eigenfunctions for the $-\lambda=36-\lambda=49-\lambda=64-\lambda=81-\lambda=100$ problem (24), (25) under different eigenvalues $\alpha=0.4, c_{3}=-1, q=0$

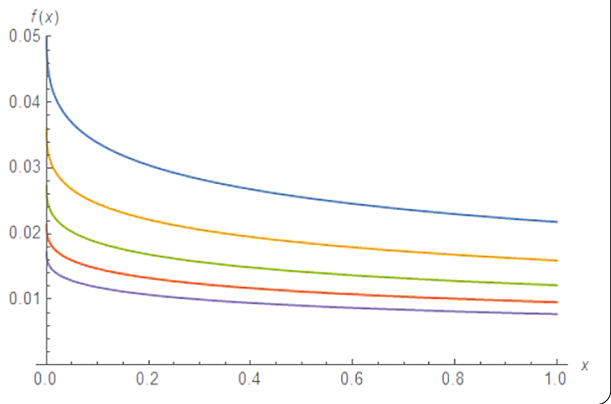

Figure 15 Comparions of eigenfunctions for the problem (24), (25) under different domains $\alpha=0.4$, $c_{3}=1, q=0$

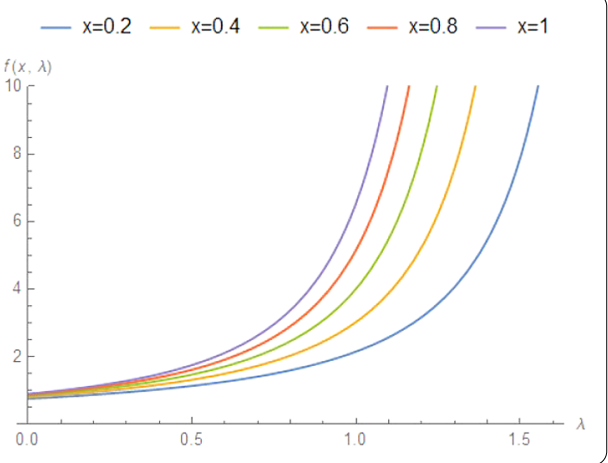

Eigenvalues of problem (24)-(25) corresponding to some specific eigenfunctions are given with different orders in Table 1 and Table 2. Finally, we compare the eigenfunctions of problems (12)-(13) and (24)-(25) with different orders in Fig. 17, Fig. 18, and Fig. 19. 
Figure 16 Comparions of eigenfunctions for the problem (24), (25) under different potentials $\lambda=36$, $\alpha=0.3, c_{3}=-1$ $-q=0-q=5-q=10-q=15-q=20$

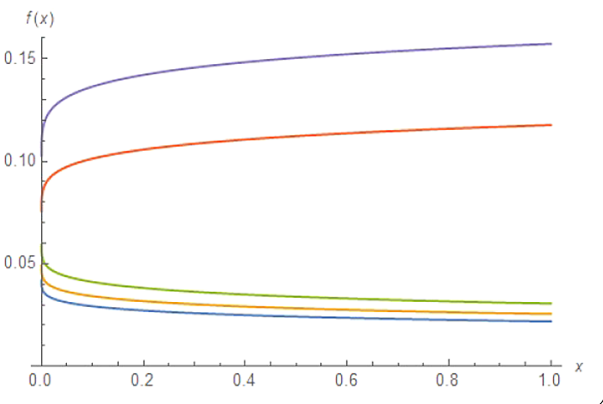

Table 1 Numerical approximations to the first four eigenvalues of problem (24)-(25) according to $E_{\delta, \theta}(z)=\sum_{k=0}^{750} \frac{z^{k}}{\Gamma(\delta k+\theta)}$. Eigenvalues correspond to eigenfunction $f(1)$

\begin{tabular}{lcccc}
\hline$\alpha$ & $\lambda_{1}$ & $\lambda_{2}$ & $\lambda_{3}$ & $\lambda_{4}$ \\
\hline 0.25 & 2.23271 & 2.25575 & 2.29206 & 2.39 \\
0.5 & 4.65311 & 4.75576 & 4.95031 & 5.76011 \\
0.6 & 6.89295 & 7.01201 & 7.08879 & 7.20048 \\
0.8 & 26.6485 & 27.057 & 27.1119 & 27.3581 \\
0.9 & -27.0582 & -21.8756 & 106.988 & 113.437 \\
\hline
\end{tabular}

Table 2 Numerical approximations to the first four eigenvalues of problem (24)-(25) according to $E_{\delta, \theta}(z)=\sum_{k=0}^{750} \frac{z^{k}}{\Gamma(\delta k+\theta)}$. Eigenvalues correspond to eigenfunction $f(0.5)$

\begin{tabular}{lcccc}
\hline$\alpha$ & $\lambda_{1}$ & $\lambda_{2}$ & $\lambda_{3}$ & $\lambda_{4}$ \\
\hline 0.25 & 2.14927 & 2.16768 & 2.19644 & 2.27351 \\
0.5 & 4.44601 & 4.51349 & 4.63887 & 5.12925 \\
0.6 & 6.6635 & 6.73779 & 6.7854 & 6.85408 \\
0.8 & 25.9274 & 26.1506 & 26.1835 & 26.3153 \\
0.9 & 103.657 & 106.885 & 109.738 & 111.407 \\
\hline
\end{tabular}

Figure 17 Comparison of eigenfunctions for the problem (12), (13)-(24), (25) $\alpha=0.3, \lambda=36$, $c_{1}=c_{3}=-1, q=0$

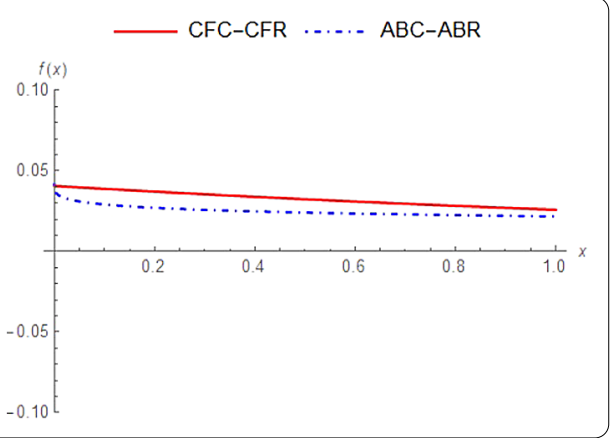

Generally, in this study, we consider fractional SL problems with different versions of new non-singular fractional operators with different versions, i.e., Caputo-Caputo, Caputo-Riemann, Riemann-Caputo, and Riemann-Riemann. We have obtained the representation of solutions of these different versions, we simulate these solutions with graphics and evaluate the solutions by means of graphics.

Also, we analyze advantages and disadvantages of these different versions. We have called SL problems (12)-(13) and (24)-(25) with Caputo-Riemann non-singular operators ${ }^{C F} L_{1}$ and ${ }^{A B} L_{1}$ respectively, problems (16)-(17) and (27)-(28) with Caputo-Caputo 


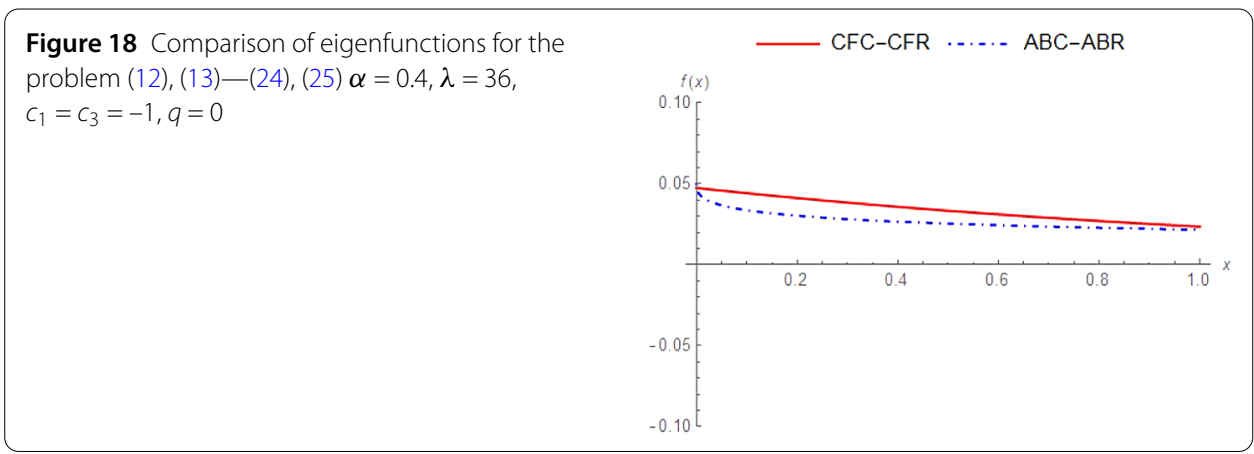

Figure 19 Comparison of eigenfunctions for the problem (12), (13)-(24), (25) $\alpha=0.5, \lambda=36$, $c_{1}=c_{3}=-1, q=0$

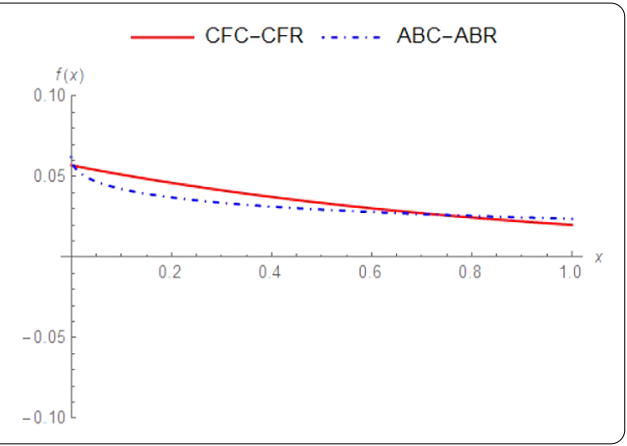

non-singular operators ${ }^{C F} L_{2}$ and ${ }^{A B} L_{2}$ respectively, problems (19)-(20) and (30)-(31) with Riemann-Caputo non-singular operators ${ }^{C F} L_{3}$ and ${ }^{A B} L_{3}$ respectively, equations (22) and (33) with Riemann-Riemann non-singular operators ${ }^{C F} L_{4}$ and ${ }^{A B} L_{4}$ respectively. Problems (12)-(13) and (24)-(25) have one initial condition and this initial condition has fractional order. Problems (16)-(17) and (27)-(28) have two initial conditions, one is fractional and one is integer order. Hence, problems (16)-(17) and (27)-(28) are more suitable in view of proving the existence and uniqueness results. Problems (19)-(20) and (30)-(31) have one initial condition and this initial condition has integer order. Equations (22) and (33) have no initial condition, thus this solution has only a nontrivial solution while $q(x)$ is not a constant.

We can observe that the eigenfunctions of problems (12)-(13), (16)-(17), and (19)-(20) converge to each other as $x$ increases in Fig. 4, additionally the eigenfunctions of problems (24)-(25), (27)-(28), and (30)-(31) show paralellism to each other in Fig. 12, and accordingly, the eigenfunctions may coincide with each other if the initial conditions are changed.

This paper may give an idea for determining the most suitable choice for defining inverse problems in fractional spectral theory.

Funding

Not applicable.

Availability of data and materials

Not applicable.

Competing interests

The authors declare to have no competing interests.

Authors' contributions

All authors read and approved the final manuscript. 


\section{Author details}

'Department of Mathematics, Firat University, Elazig, Turkey. ${ }^{2}$ Department of Mathematics and Computer Sciences, Faculty of Arts and Sciences, Cankaya University, Ankara, Turkey. ${ }^{3}$ Institute of Space Sciences, Magurele-Bucharest, Romania.

\section{Publisher's Note}

Springer Nature remains neutral with regard to jurisdictional claims in published maps and institutional affiliations.

\section{Received: 24 July 2018 Accepted: 15 September 2018 Published online: 03 October 2018}

\section{References}

1. Caputo, M., Fabrizio, M.: A new definition of fractional derivative without singular kernel. Prog. Fract. Differ. Appl. 1(2), $1-13(2015)$

2. Atangana, A., Baleanu, D.: New fractional derivatives with non-local and nonsingular kernel: theory and application to heat transfer model. Therm. Sci. 20(2), 763-769 (2016)

3. Atangana, A., Baleanu, D.: Caputo-Fabrizio derivative applied to groundwater flow within confined aquifer. J. Eng. Mech. 143(5), D4016005 (2017)

4. Gómez-Aguilar, J.F., Morales-Delgado, V.F., Taneco-Hernández, M.A., Baleanu, D., Escobar-Jiménez, R.F., Al Qurashi, M.M.: Analytical solutions of the electrical RLC circuit via Liouville-Caputo operators with local and non-local kernels. Entropy 18(8), 402 (2016)

5. Alsaedi, A., Baleanu, D., Etemad, S., Rezapour, S.: On coupled systems of time-fractional differential problems by using a new fractional derivative. J. Funct. Spaces 2016, Article ID 4626940 (2016)

6. Jarad, F., Uğurlu, E., Abdeljawad, T., Baleanu, D.: On a new class of fractional operators. Adv. Differ. Equ. 1, 247 (2017)

7. Uğurlu, E., Baleanu, D., Taş, K.: On the solutions of a fractional boundary value problem. Turk. J. Math. 42(3), 1307-1311 (2018)

8. Owolabi, K.M., Atangana, A.: Numerical approximation of nonlinear fractional parabolic differential equations with Caputo-Fabrizio derivative in Riemann-Liouville sense. Chaos Solitons Fractals 99, 171-179 (2017)

9. Atangana, A., Alqahtani, R.T.: Numerical approximation of the space-time Caputo-Fabrizio fractional derivative and application to groundwater pollution equation. Adv. Differ. Equ. 2016(1), 156 (2016)

10. Al-Refai, M., Abdeljawad, T.: Analysis of the fractional diffusion equations with fractional derivative of non-singular kernel. Adv. Differ. Equ. 1, 315 (2017)

11. Abdeljawad, T.: Fractional operators with exponential kernels and a Lyapunov type inequality. Adv. Differ. Equ. 1, 313 (2017)

12. Carvalho, A.R.: Fractional dynamics of an infection model with time-varying drug exposure. J. Comput. Nonlinear Dyn. 13, 090904 (2018)

13. Carvalho, A.R., Pinto, C.M., Baleanu, D.: HIV/HCV coinfection model: a fractional-order perspective for the effect of the HIV viral load. Adv. Differ. Equ. 2018(1), 2 (2018)

14. Pinto, C.M.: Novel results for asymmetrically coupled fractional neurons. Acta Polytech. Hung. 14(1), 177-189 (2017)

15. Pinto, C.M.: Strange dynamics in a fractional derivative of complex-order network of chaotic oscillators. Int. J. Bifurc. Chaos 25(01), $1550003(2015)$

16. Baskonus, H.M., Mekkaoui, T., Hammouch, Z., Bulut, H.: Active control of a chaotic fractional order economic system Entropy 17(8), 5771-5783 (2015)

17. Baskonus, H.M., Bulut, H.: On the numerical solutions of some fractional ordinary differential equations by fractional Adams-Bashforth-Moulton method. Open Math. 13(1), 547-556 (2015)

18. Baskonus, H.M., Bulut, H.: Regarding on the prototype solutions for the nonlinear fractional-order biological population model. In: AlP Conference Proceedings, vol. 1738, p. 290004. AIP, New York (2016)

19. Baskonus, H.M., Hammouch, Z., Mekkaoui, T., Bulut, H.: Chaos in the fractional order logistic delay system: circuit realization and synchronization. In: AIP Conference Proceedings, vol. 1738, p. 290005. AIP, New York (2016)

20. Ravichandran, C., Jothimani, K., Baskonus, H.M., Valliammal, N.: New results on nondensely characterized integrodifferential equations with fractional order. Eur. Phys. J. Plus 133(3), 109 (2018)

21. Dokuyucu, M.A., Celik, E., Bulut, H., Baskonus, H.M.: Cancer treatment model with the Caputo-Fabrizio fractional derivative. Eur. Phys. J. Plus 133(3), 92 (2018)

22. Esen, A., Sulaiman, T.A., Bulut, H., Baskonus, H.M.: Optical solitons to the space-time fractional (1 + 1)-dimensional coupled nonlinear Schrödinger equation. Optik 167, 150-156 (2018)

23. Bulut, H., Kumar, D., Singh, J., Swroop, R., Baskonus, H.M.: Analytic study for a fractional model of HIV infection of CD4+TCD4+T lymphocyte cells. Math. Nat. Sci. 2(1), 33-43 (2018)

24. Yavuz, M., Ozdemir, M.N., Baskonus, H.M.: Solution of fractional partial differential equation using the operator involving non-singular kernel. Eur. Phys. J. Plus 133(215), 1-12 (2018)

25. Abdeljawad, T., Baleanu, D.: On fractional derivatives with exponential kernel and their discrete versions. Rep. Math. Phys. 80(1), 11-27 (2017)

26. Abdeljawad, T., Baleanu, D.: Integration by parts and its applications of a new nonlocal fractional derivative with Mittag-Leffler nonsingular kernel. J. Nonlinear Sci. Appl. 10, 1098-1107 (2017)

27. Sun, H., Hao, X., Zhang, Y., Baleanu, D.: Relaxation and diffusion models with non-singular kernels. Phys. A, Stat. Mech. Appl. 468, 590-596 (2017)

28. Gómez-Aguilar, J.F., Atangana, A.: New insight in fractional differentiation: power, exponential decay and Mittag-Leffler laws and applications. Eur. Phys. J. Plus 132(1), 13 (2017)

29. Gómez-Aguilar, J.F., Atangana, A., Morales-Delgado, V.F.: Electrical circuits RC, LC, and RL described by Atangana-Baleanu fractional derivatives. Int. J. Circuit Theory Appl. 45(11), 1514-1533 (2017)

30. Abro, K.A. Memon, A.A., Uqaili, M.A.: A comparative mathematical analysis of RL and RC electrical circuits via Atangana-Baleanu and Caputo-Fabrizio fractional derivatives. Eur. Phys. J. Plus 133(3), 113 (2018) 
31. Sheikh, N.A., Ali, F., Saqib, M., Khan, I., Jan, S.A.A.: A comparative study of Atangana-Baleanu and Caputo-Fabrizio fractional derivatives to the convective flow of a generalized Casson fluid. Eur. Phys. J. Plus 132(1), 54 (2017)

32. Sheikh, N.A., Ali, F., Saqib, M., Khan, I., Jan, S.A.A., Alshomrani, A.S., Alghamdi, M.S.: Comparison and analysis of the Atangana-Baleanu and Caputo-Fabrizio fractional derivatives for generalized Casson fluid model with heat generation and chemical reaction. Results Phys. 7, 789-800 (2017)

33. Saad, K.M.: Comparing the Caputo, Caputo-Fabrizio and Atangana-Baleanu derivative with fractional order: fractional cubic isothermal auto-catalytic chemical system. Eur. Phys. J. Plus 133(3), 94 (2018)

34. Gómez-Aguilar, J.F., Yépez-Martínez, H., Calderón-Ramón, C., Cruz-Orduña, I., Escobar-Jiménez, R.F., Olivares-Peregrino, V.H.: Modeling of a mass-spring-damper system by fractional derivatives with and without a singular kernel. Entropy 17(9), 6289-6303 (2015)

35. Klimek, M., Agrawal, O.P.: On a regular fractional Sturm-Liouville problem with derivatives of order in $(0,1)$. In: Carpathian Control Conference (ICCC), 2012 13th International pp. 284-289. IEEE Comput. Soc., Los Alamitos (2012)

36. Bas, E., Metin, F.: Fractional singular Sturm-Liouville operator for Coulomb potential. Adv. Differ. Equ. 1, 300 (2013)

37. Bas, E.: The inverse nodal problem for the fractional diffusion equation. Acta Sci., Technol. 37(2), 251 (2015)

38. Zayernouri, M., Karniadakis, G.E.: Fractional Sturm-Liouville eigen-problems: theory and numerical approximation. J. Comput. Phys. 252, 495-517 (2013)

39. Khosravian-Arab, H., Dehghan, M., Eslahchi, M.R.: Fractional Sturm-Liouville boundary value problems in unbounded domains: theory and applications. J. Comput. Phys. 299, 526-560 (2015)

40. Dehghan, M., Mingarelli, A.B.: Fractional Sturm-Liouville eigenvalue problems, II (2017). arXiv:1712.09894. arXiv preprint

41. Podlubny, I.: Fractional Differential Equations: An Introduction to Fractional Derivatives, Fractional Differentia Equations, to Methods of Their Solution and Some of Their Applications. vol. 198. Elsevier, Amsterdam (1998)

\section{Submit your manuscript to a SpringerOpen ${ }^{\circ}$ journal and benefit from:}

- Convenient online submission

Rigorous peer review

- Open access: articles freely available online

- High visibility within the field

- Retaining the copyright to your article

Submit your next manuscript at $>$ springeropen.com 